\title{
Christopher Rowland, (ed.), The Cambridge Companion to Liberation Theology
}

Cambridge, Cambridge University Press, 2007, 318 p.

\section{Michael Löwy}

\section{CpenEdition} Journals

Édition électronique

URL : http://journals.openedition.org/assr/22502

DOI : $10.4000 /$ assr.22502

ISSN : 1777-5825

Éditeur

Éditions de l'EHESS

Édition imprimée

Date de publication : 31 décembre 2010

Pagination : 9-242

ISBN : 9782713223013

ISSN : 0335-5985

Référence électronique

Michael Löwy, "Christopher RowLAnd, (ed.), The Cambridge Companion to Liberation Theology », Archives de sciences sociales des religions [En ligne], 152 I octobre-décembre 2010, document 152-103, mis en ligne le 12 mai 2011, consulté le 21 septembre 2020. URL : http://journals.openedition.org/ assr/22502 ; DOI : https://doi.org/10.4000/assr.22502

Ce document a été généré automatiquement le 21 septembre 2020.

(c) Archives de sciences sociales des religions 


\title{
Christopher Rowland, (ed.), The Cambridge Companion to Liberation Theology
}

Cambridge, Cambridge University Press, 2007, 318 p.

\author{
Michael Löwy
}

\section{RÉFÉRENCE}

Christopher Rowland, (ed.), The Cambridge Companion to Liberation Theology,

Cambridge, Cambridge University Press, 2007, 318 p.

1 L'entrée de la théologie de la libération dans cette prestigieuse collection de livres publiées par l'université de Cambridge est en soi un événement. Il s'agit en fait d'un recueil publié en 1999, qui fait, en 2007, l'objet d'une réédition actualisée et élargie. Les textes sont regroupés en trois sections intitulées «La théologie de la libération contemporaine», «Aspects de la théologie de la libération» et "Analyse et Critique». Comme c'est souvent le cas, ces grands chapitres ne veulent pas dire grand-chose, chacun des textes aurait pu trouver sa place dans l'une ou dans l'autre partie.

Le recueil est ouvert par des documents qui annoncent la problématique du livre: une préface et une introduction par l'éditeur duvolume, Christopher Rowland, Professeur d'exégèse de l'Écriture sainte à l'Université d'Oxford, et un essai par Gustavo Gutierrez, le fondateur (péruvien) de la théologie de la libération. Le contraste entre ces deux démarches est frappant, et mériterait en soi une analyse sociologique... L'introduction de Rowland rappelle quelques aspects essentiels de la théologie de la libération: il s'agit d'une nouvelle façon de faire de la théologie, à partir de la perspective des pauvres et des marginalisés, qui se situe dans le cadre du magistère de l'Église catholique. Il ajoute - ce qui est inhabituel - qu'on peut trouver des antécédents historiques dans des lectures radicales (plutôt d'origine protestante) de la Bible, comme celles de Gerrard Winstanley (XVII ${ }^{e}$ siècle) et William Blake (XIX ${ }^{e}$ siècle). On constate que 
le professeur d'Oxford a un authentique intérêt et une grande sympathie pour la théologie de la libération, mais sa compréhension du phénomène est limitée par une lecture typiquement anglo-américaine - un «point de vue du Nord» diraient les théologiens latino-américains. Ainsi, dans la préface, il propose de remplacer le terme de théologie de la libération par celui de «théologie contextuelle» - un terme qui désigne le dialogue entre le contexte social et l'Écriture - comme si toute théologie, depuis Augustin, comme le reconnaît Rowland lui-même quelques lignes plus loin, n'était pas en rapport avec un contexte social! Il ajoute, toujours dans cette préface, que la théologie de la libération n'est pas tellement nouvelle, puisqu'elle plonge ses racines dans l'importance attribuée par le christianisme à une vie de prière et d'engagement pour le voisin...

3 De même, dans son Introduction, Rowland définit la théologie de la libération comme un mouvement de chrétiens «engagés pour l'amélioration (committed to the betterment) de la condition des pauvres». Ce qui disparaît dans cette réflexion, c'est tout simplement l'essentiel de la théologie de la libération: la libération, comme action collective des pauvres.

4 L'essai de Gustavo Gutierrez relève d'une tout autre tonalité. Son point de départ est «l'irruption des pauvres», qui commencent à se percevoir eux-mêmes comme les sujets de leur propre histoire, capables de prendre leur destin en leurs propres mains. Le rôle des chrétiens n'est pas d'«améliorer» le sort des pauvres mais de participer au «processus de libération en Amérique Latine» - comme l'ont fait Monseigneur Oscar Romero (assassiné en 1980) et tant d'autres martyrs - une libération qui est à la fois sociale, humaine et morale, et dont l'objectif est de construire une société nouvelle et fraternelle, annonciatrice du Royaume de Dieu.

5 Au-delà des évidentes différences de contenu, le contraste entre le style, la problématique, la «musique» des deux textes, est évident. Ils ne se situent pas sur le même terrain sociohistorique: "Oxbridge» d'une part, la réalité socioreligieuse de l'Amérique Latine de l'autre... On retrouve cet écart dans la plupart des essais du recueil, même s'ils apportent des analyses intéressantes, par exemple sur la théologie noire (Edward Antonio), sur la théologie féministe (Mary Grey), la Bible (Gerald West) ou la théologie politique (Oliver O'Donovan).

6 Prenons, pour illustrer ces difficultés, l'article sur les idées économiques de la théologie de la libération de Valpy Fitgerald, également professeur à Oxford. Il observe, à juste titre, que la théologie de la libération se distingue de la doctrine sociale de l'Église des encycliques papales par la conviction qu'il ne suffit pas de corriger les erreurs ou les excès du capitalisme, mais qu'il faut le remplacer par une autre civilisation, fondée sur la satisfaction des besoins essentiels des pauvres. Il constate aussi que, sans adhérer au matérialisme historique, les théologiens latino-américains associent la critique marxiste du fétichisme de la marchandise avec celle, biblique, de l'idolâtrie (il est dommage que Hugo Assmann, le principal représentant de cette critique théologique du capitalisme, ne soit pas mentionné). Mais, en dernière analyse, l'auteur regrette que la théologie de la libération ait négligé des «questions centrales de la théorie économique moderne», telles que «les incitations aux producteurs privés», ou «l'économie du bien-être social» (welfare economics). Le professeur Fitzgerald semble considérer l'économie politique anglo-américaine établie comme «la théorie économique moderne»; ce qu'il met sur le compte d'une négligence de la part de la théologie de la libération est tout simplement son refus d'accepter les termes du débat 
économique institutionnel, celui qui va de soi dans les départements d'économie de Chicago ou d'Oxford.

7 On trouve une démarche analogue dans la conclusion de l'essai - par ailleurs intéressant - sur la globalisation de la théologie de la libération d'Ivan Petrella, qui conseille à la théologie de la libération de dépasser la condamnation du "capitalisme» en général, pour s'intéresser à «la façon par laquelle les détails légaux des diverses formes de capitalismes réellement existants affectent la distribution des ressources de la société».

Un autre exemple frappant d'une posture qui reste enfermée dans la problématique des pays $\mathrm{du}$ «Nord» est la discussion sur le marxisme. Christopher Rowland, dans sa préface à la seconde édition, prétend que la théologie de la libération n'a jamais été sérieusement influencée par le marxisme, même si sur certaines questions «il y a des parallélismes entre les deux». Cette hypothèse peu vraisemblable est en fait rejetée par plusieurs auteurs - Charles Villa-Vicencio, Bastiaan Wielenga - et surtout par Denis Turner, professeur à l'Université de Yale, dans son essai sur «Le marxisme, la théologie de la libération et la voie de la négation». Après une discussion intéressante sur les instructions vaticanes contre la théologie de la libération, Turner reproche aux théologiens latino-américains de ne pas s'intéresser à l'athéisme de Marx, qu'il compare à la tradition de théologie négative de la mystique occidentale, de Maître Eckhardt à saint Jean de la Croix. Il manque à la théologie de la libération, insiste-t-il, une théologie négative de l'absence de Dieu, une théologie apophatique - Dieu ne peut pas être décrit par le langage humain - qui présente des analogies avec l'athéisme marxien. Outre le caractère très discutable de cette analogie, le curieux reproche de Turner ne prend pas en considération les raisons pour lesquelles la théologie de la libération ne s'intéresse pas à la question de l'athéisme: 1-les couches populaires en Amérique Latine sont, dans leur écrasante majorité, croyantes; 2-pour la théologie de la libération, l'adversaire du christianisme n'est pas l'athéisme, mais l'idolâtrie (du pouvoir, de l'argent, de la marchandise).

Certains des travaux ici publiés échappent cependant à ce regard «occidental». C'est le cas de l'article de Bastiaan Wielenga, un théologien hollandais vivant en Inde depuis plusieurs décennies, qui analyse la théologie de la libération en Asie; il est d'ailleurs un des rares auteurs de ce recueil à s'intéresser de près à l'écologie. Ou celui d'Andrew Dawson sur les communautés ecclésiales de base au Brésil: un travail bien documenté, mais qui va un peu trop vite en affirmant que, depuis 1983, l'orthodoxie vaticane est devenue hégémonique dans l'Église brésilienne, dont les activités pastorales se sont dirigées à nouveau vers les classes moyennes et l'élite bourgeoise. Enfin, c'est le cas du théologien jésuite américain Peter Hebblethwaite, dont l'essai sur le conflit entre Rome et la théologie de la libération - rédigé en 1994, peu avant sa mort - est une belle étude critique des documents du Vatican. Selon l'auteur, l'Instruction de 1984 sur «certains aspects de la théologie de la libération» est un des documents les plus radicaux publiés par le Vatican, parce que, dans leur anxiété de réfuter la théologie de la libération, ses auteurs ont été obligés d'emprunter ses habits. Cela vaut moins pour la Deuxième Instruction (1986), rédigée par Jean-Paul II, qui interprète l'option préférentielle pour les pauvres en termes de charité chrétienne. À partir de ce moment, observe le jésuite américain, les attaques théologiques sont remplacées par des mesures administratives: «nomination d'évêques réactionnaires, intense pression sur les supérieurs religieux 
pour qu'ils expulsent les théologiens de la libération (...), encouragement aux mouvements de droite hostiles à la théologie de la libération».

10 En conclusion, un recueil inégal, mais qui contient des contributions importantes, et qui témoigne de l'intérêt des milieux universitaires anglo-américains pour la théologie de la libération. 